\title{
Qualidade de vida de doentes esofagectomizados: adenocarcinoma versus carcinoma epidermoide
}

\section{Quality of life of esophagectomized patients: adenocarcinoma versus squamous cell carcinoma}

\author{
Maricilda Regina Pereira'; Luiz Roberto Lopes, TCBC-SP²; Nelson Adami Andreollo TCBC-SP33
}

\author{
RES U M O
}

\begin{abstract}
Objetivo: Avaliar e comparar a qualidade de vida de pacientes esofagectomizados para tratamento de adenocarcinoma da junção esofagogástrica e de carcinoma epidermoide. Métodos: estudo transversal no pós-operatório de doentes esofagectomizados por adenocarcinoma da junção esofagogástrica (Adenoca) e carcinoma epidermóide (CEC), empregando o questionário SF-36 aplicado em 24 pacientes (10 por Adenoca e 14 por CEC), a partir do $5^{\circ}$ mês de pós-operatório, incluindo os sintomas clínicos e a variação de peso. Resultados: A avaliação da QV mostrou melhor resultado de capacidade funcional $(p=0,018)$ para o grupo Adenoca. Houve correlação entre os domínios "saúde mental" e "limitação por aspectos emocionais" ( $p=0,003)$ e entre "dor" e "limitação por aspectos físicos" ( $p=0,003)$ nos dois tipos histológicos. A perda de peso foi maior nos esofagectomizados por Adenoca $(45,9 \mathrm{Kg})$, sem diferença significativa entre o IMC atual ( $p>0,66)$. A disfagia foi relatada por 83,3\% dos pacientes, a anorexia por 58,3\%, a dificuldade de mastigação por $42 \%$, a náuseas e os vômitos por $41,7 \%$ e a diarréia por $29,2 \%$, sem correlação com a QV relatada $(p>0,05)$. Conclusão: $O$ escore mais alto para capacidade funcional indica que o paciente com Adenoca foi capaz de realizar todo tipo de atividade física, incluindo as mais vigorosas em um nível maior que o paciente com CEC. Alguns sintomas persistiram no pósoperatório, porém não interferiram na qualidade de vida dos pacientes.
\end{abstract}

Descritores: Adenocarcinoma. Carcinoma de células escamosas. Qualidade de vida. Esofagectomia. Transtornos de deglutição.

\section{INTRODUÇÃO}

$\mathrm{O}$ carcinoma epidermóide de células escamosas (CEC) foi o principal alvo da atenção dos estudos sobre o câncer esofágico ${ }^{1}$. Entretanto, aumento da incidência do adenocarcinoma da junção esofagogástrica (Adenoca) tem sido relatado nas últimas quatro décadas².

O CEC possui etiologia fortemente relacionada ao tabagismo e ao etilismo, e o adenocarcinoma da junção esofagogástrica está associado ao esôfago de Barrett e ao refluxo gastroesofagiano ${ }^{3}$. Há evidências sugerindo que a obesidade está positivamente associada ao risco de desenvolver o Adenoca esofágico, enquanto que a desnutrição tem sido associada ao $\mathrm{CEC}^{4}$.

Existem análises comparativas da qualidade de vida de pacientes esofagectomizados segundo alguns aspectos cirúrgicos, tais como a comparação entre a ressecção limitada trans-hiatal com a extensa ressecção transtorácica5; entre a reconstrução com o tubo gástrico e com a interposição com o cólon ${ }^{6}$, a comparação entre o trata- mento curativo após a esofagectomia e o tratamento paliativo ${ }^{7,8}$ ou mesmo a comparação entre os domínios da qualidade de vida com os sintomas (físicos e emocionais) e fatores relacionados à doença e à operação ${ }^{9}$. Não foram encontradas na literatura consultada as possíveis diferenças existentes entre a qualidade de vida dos doentes esofagectomizados por Adenoca da transição esofagogástrica com os esofagectomizados por CEC.

De Boer et al. compararam a qualidade de vida de esofagectomizados por adenoca após três anos de ressecção trans-hiatal ou transtorácica ${ }^{5}$. Por outro lado, Rosmolen et al., em estudo longitudinal, analisaram a qualidade de vida entre sobreviventes de longo período ${ }^{8}$ (mínimo de dois anos) após ressecção transmediastinal e reconstrução com tubo gástrico, de 100 doentes, também em doentes prioritariamente com Adenoca. De Boer et al. em outro estudo longitudinal, incluíram $67 \%$ da amostra total estudada de Adenoca e $28 \%$ de CEC 9 .

Nos primeiros seis meses de pós-operatório o doente experimenta, durante um-período de tempo variá-

Trabalho realizado na Disciplina de Moléstias do Aparelho Digestivo e Gastrocentro do Departamento de Cirurgia da Faculdade de Ciências Médicas da Universidade Estadual de Campinas - UNICAMP - Campinas - SP-BR.

1. Nutricionista e Pós-Graduanda do Curso de Pós-Graduação em Ciências da Cirurgia da Faculdade de Ciências Médicas da UNICAMP- SP-BR;

2. Professor Livre-Docente da Disciplina de Moléstias do Aparelho Digestivo e Gastrocentro do Departamento de Cirurgia da Faculdade de Ciências Médicas da UNICAMP- SP-BR; 3. Professor Titular da Disciplina de Moléstias do Aparelho Digestivo e Gastrocentro do Departamento de Cirurgia da Faculdade de Ciências Médicas da UNICAMP- SP-BR. 
vel, sintomas indesejáveis, como a pouca aceitação alimentar, náuseas, a contínua perda de peso, a sensação de fraqueza, a diarreia, a disfagia e dor ${ }^{10}$ e uma qualidade de vida que pode ser muito prejudicada, retornando aos níveis pré-operatórios ${ }^{7,11}$. Assim, sendo possível melhor avaliar e investigar estes sintomas, certamente eles poderão ser minimizados, mediante orientações e tratamentos, melhorando a qualidade de vida do doente.

O objetivo deste trabalho foi avaliar e comparar a qualidade de vida de doentes esofagectomizados por apresentarem adenocarcinoma da junção esofagogástrica e carcinoma epidermóide de células escamosas, a partir do quinto mês de pós-operatório, empregando o questionário genérico SF-36, além de investigar a variação de peso corpóreo, a presença de disfagia, anorexia, náuseas, vômitos, diarreia e constipação e relacioná-los à qualidade de vida .

\section{MÉTODOS}

Foram realizadas análises transversais de 24 pacientes esofagectomizados no período de setembro de 2004 a agosto de 2008, em decorrência de câncer de esôfago ou da cárdia, a partir do quinto mês de pós-operatório. As operações foram realizadas pelo Grupo de Cirurgia de Esôfago, Estômago e Duodeno do Hospital das Clínicas da Faculdade de Ciências Médicas da Universidade de Campinas (UNICAMP), conforme a técnica padronizada transmediastinal, seguido de esofagogastroplastia com tubo gástrico, anastomose esofagogástrica cervical, piloromiotomia e jejunostomia para alimentação em todos os casos.

Esta pesquisa foi desenvolvida atendendo às exigências da Resolução MS/CNS nº 196, de 10 de outubro de 1996, e foi aprovada pelo Comitê de Ética e Pesquisa da FCM-UNICAMP, conforme protocolo n 505/2003 em 20/07/2004.

Como critério de inclusão, selecionaram-se os pacientes que não estavam em tratamento de quimio ou radioterapia e não apresentavam metástases no momento do estudo. Nenhum paciente apresentou dificuldade de verbalização ou de memória para responder às questões solicitadas e todos concordaram em assinar o Termo de Consentimento Livre Esclarecido.

Não foram incluídos os pacientes esofagectomizados por doença benigna, pós-operatório imediato ou em tempo inferior a cinco meses, acamado e os que não puderam comparecer ao ambulatório para as avaliações.

Os pacientes foram convidados, via assistente social, para comparecerem no ambulatório na data estabelecida para responderem ao questionário SF-36 e para o exame físico, registro da história clínica, dos dados sócio-econômicos e culturais e dos sintomas clínicos.
Os dados analisados foram: idade, sexo, estado civil, raça, escolaridade, tipo histológico, o tempo decorrido entre a operação e a entrevista, peso e estatura para o cálculo do índice de massa corporal (IMC) obtidos em balança plataforma, com o indivíduo descalço e trajando roupas leves. Os resultados do IMC foram avaliados segundo a Organização Mundial da Saúde ${ }^{12}$ para os indivíduos adultos e segundo Lipschitz ${ }^{13}$ para idosos.

A perda de peso foi calculada pela análise da porcentagem de adequação entre o peso habitual ou usual do paciente antes da operação e o peso atual. A presença de disfagia, dificuldade de mastigação, anorexia, náuseas, vômitos, diarreia e constipação foram investigados durante a entrevista com o paciente.

A qualidade de vida dos pacientes foi avaliada através do questionário "Medical Outcomes Study 36-Item Short Form Health Survey" (SF-36) utilizado na prática clínica em geral, em pesquisas, avaliação de políticas de saúde e análises populacionais, para medir qualidade de vida geral. Este questionário foi desenvolvido por Ware e Sherbourne ${ }^{14}$ e traduzido e validado para o português por Ciconelli et al. ${ }^{15}$.

O SF-36 é composto por 11 questões com subítens que totalizam 36 perguntas distribuídas por oito domínios. Estes domínios são agrupados em dois grandes componentes: o físico e o mental.

O componente físico envolve os domínios capacidade funcional, aspectos físicos, dor e estado geral de saúde, enquanto que o componente mental compreende os domínios saúde mental, vitalidade, aspectos emocionais e sociais. Os resultados obtidos serão comparados com os de Aaronson et al. ${ }^{16}$

Os resultados obtidos no questionário de avaliação da qualidade de vida SF-36 mais perto de 100 indicam melhor resultado e pior quanto mais perto de zero.

Devido à característica da doença, o tamanho da amostra foi estabelecido segundo a disponibilidade de pacientes no período levantado, a ocorrência de óbitos, a perda de seguimento e ponderação sobre os aspectos práticos, tais como limitações de tempo e questões de estatística. Foram utilizadas as frequências absolutas (n) e relativas (\%) para as variáveis de natureza qualitativa nominal, e a média e desvio-padrão para as variáveis de natureza quantitativa e para a descrição dos valores encontrados.

O teste t de Student foi aplicado para o estudo das médias de IMC de cada tipo histológico e para as comparações dos resultados obtidos nos domínios: "Dor", "Estado Geral de Saúde" e "Saúde Mental" e o teste MannWhitney para os domínios: "Capacidade Funcional", "Limitação por aspectos físicos", "Vitalidade", "Aspectos Sociais" e "Limitação por Aspectos Emocionais".

A busca por associação entre o tempo decorrido após a operação até a entrevista e os resultados obtidos na avaliação da qualidade de vida foi realizada pelo teste de correlação de Pearson. Além disso, foi analisado 
se o tempo decorrido da operação até a entrevista influenciou nos resultados da qualidade de vida dos pacientes entrevistados. A comparação entre o tempo médio obtido para cada tipo histológico foi realizada pelo teste de Mann-Whitney.

A associação de anorexia, disfagia, náuseas/vômitos, hábitos intestinais e o tipo histológico de câncer foram realizados pelo teste de Fisher. Foram considerados significativos os valores de " $p$ " inferiores a 0,05.

\section{RESULTADOS}

Os 24 participantes deste estudo foram na maioria homens $(91,7 \%)$, da raça branca $(70,8 \%)$, casados (83,3\%), média de idade de 58,8 anos, mínimo de 44 e máximo 83 anos. Cabe destacar que 37,5\% são idosos, ou seja, com idade superior a 60 anos. Quanto à escolaridade, a média encontrada para esta amostra foi de 4,9 anos estudados (Tabela 1).

Os pacientes elegíveis para a pesquisa foram distribuídos em dois grupos segundo o tipo histológico: o Adenoca $(n=10)$ e o CEC $(n=14)$. Todos foram avaliados com um mínimo de cinco meses de pós-operatório e o máximo de oito anos, 11 meses e quatro dias. O tempo médio de pós-operatório para os participantes da pesquisa foi dois anos, quatro meses e 26 dias e, segundo o tipo

Tabela 1 - Características da população de pacientes estudada.

\begin{tabular}{lrc}
\hline Características & N $(\%)$ \\
\hline Sexo & 22 & $(91,6)$ \\
$\mathrm{M}$ & 2 & $(8,4)$ \\
$\mathrm{F}$ & & \\
Idade (anos) & 15 & $(62,5)$ \\
$<60$ & 4 & $(16,7)$ \\
$60-69$ & 5 & $(20,8)$ \\
70 a 83 & & \\
Cor da Pele & 17 & $(70,8)$ \\
Brancos & 7 & $(29,2)$ \\
Não brancos & & \\
Tipo Histológico do tumor & 10 & $(41,7)$ \\
Adenocarcinoma de esôfago ou cárdia & 14 & $(58,3)$ \\
Carcinoma epidermoide de células escamosas & & \\
Estado Conjugal & 20 & $(83,4)$ \\
Casado ou amasiado & 4 & $(16,6)$ \\
Solteiro/ desquitado/ divorciado & & \\
Escolaridade & 2 & $(8,3)$ \\
Analfabeto & 11 & $(45,8)$ \\
Até 4 anos de estudo & 3 & $(12,5)$ \\
Até 7 anos de estudo & 1 & $(4,17)$ \\
Acima de 7 anos de estudo & 1 & $(4,17)$ \\
Superior completo & $6(25)$ \\
Não declarado & & \\
\hline
\end{tabular}

histológico, nos casos de Adenoca o tempo médio foi um ano, oito meses e cinco dias e para o CEC, dois anos, dez meses e 31 dias. Entretanto, esta diferença de tempo entre os dois tipos histológicos não foi significativa ( $p=0.8168$ ).

A distribuição das variáveis de qualidade de vida e tempo decorrido da operação pode ser vista na figura 1.

A tabela 2 demonstra os valores dos componentes dos escores SF-36 obtidos e relacionados ao tempo decorrido de operação.

A média de porcentagem de perda de peso ( $\mathrm{Ta}$ bela 3) foi maior entre os pacientes com Adenoca (16,84\%). Entretanto, a média do IMC atual para do grupo adenoca foi 21,5 (SD-2,7) e para o grupo CEC foi 21,0 (SD-2,8), diferença não significativa entre os dois resultados $(p=0,6602)$.

Ao comparar a qualidade de vida dos dois tipos histológicos do câncer esofágico houve diferença significativa no domínio "Capacidade Funcional", e igualdade em todos os outros domínios.

Analisando as respostas coletadas nos oito componentes do SF-36 que foram apresentadas na tabela 4 pode-se afirmar que há diferença entre os dois tipos histológicos para o domínio "Capacidade Funcional" $(p=0,017)$ e que, para todos os outros domínios não se rejeita a igualdade, e nos domínios "Dor", "Vitalidade", "Saúde Mental" e "Limitação por Aspectos Emocionais", existe uma forte evidência de igualdade entre os tipos histológicos.

O resultado obtido no domínio "Aspectos Sociais" mostra uma média um pouco diferenciada entre os dois tipos histológicos, porém não foi considerado significativo ( $p=0,2570)$, o mesmo ocorreu para "Estado Geral de Saúde" ( $p=0,3591)$, "Limitação por Aspectos físicos" $(p=0,3614)$ e "Limitação por Aspectos Emocionais" $(p=0,7317)$.

Os domínios "Limitações por Aspectos Emocionais" e "Limitação por Aspectos Físicos" têm o maior desvio padrão entre todas as categorias, sendo que a média do componente "Limitação por Aspectos Emocionais" fol 20 pontos maior que a média da variável "Limitação por Aspectos Físicos".

A análise da correlação entre a variável "Saúde Mental" e "Limitação por Aspectos Emocionais" tem como resultado $p=0,587$, com um $p$-valor de 0,003, e uma forte evidência de que há uma relação entre as duas variáveis. Resultados semelhantes foram obtidos nas variáveis "Limitação por Aspectos Físicos" e "Dor" ; $(p=0,586$ e o $p$-valor de 0,003$)$. $E$, analisando a correlação destas variáveis entre os pacientes com Adenoca e CEC separadamente, a correlação foi ainda maior.

Os resultados obtidos com os 24 doentes estudados após a aplicação do SF-36 estão inseridos na tabela 4. A análise estatística empregando o teste exato de Fischer mostrou que há diferenças estatísticas significantes no domínio dor $(p=0,032)$ para o grupo Adenoca e, na capacidade funcional $(p=0,003)$, limitação por aspectos físicos 


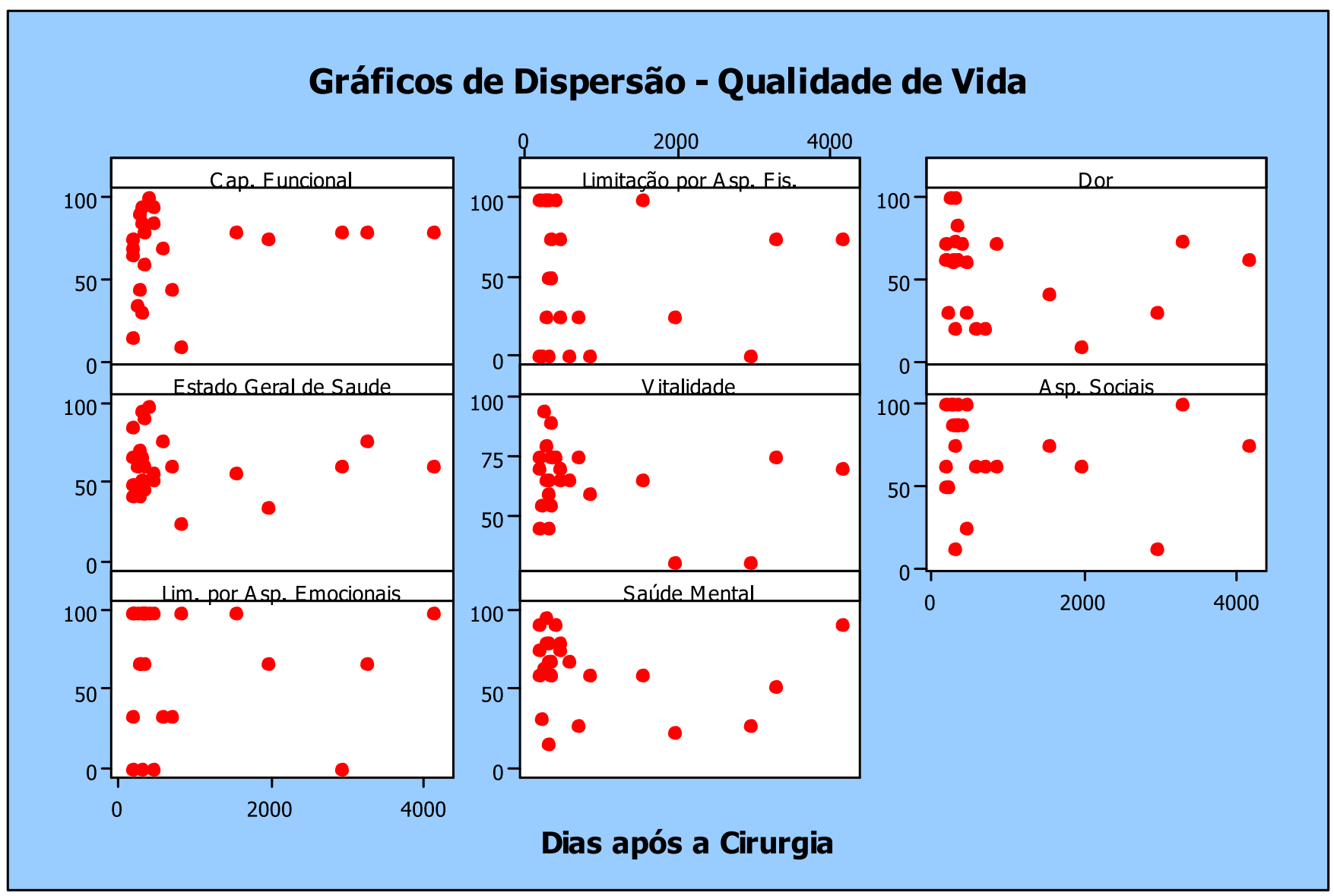

Figura 1 - Correlação entre as variáveis da qualidade de vida e o tempo decorrido da operação até a entrevista.

$(p=0,027)$ e nos aspectos sociais $(p=0,0468)$ para o grupo CEC.

A análise dos dados clínicos mostra que não houve associação entre a disfagia, anorexia, náuseas, vômitos, diarreia e constipação e o tipo histológico (Tabela 5). A disfagia foi referida por $83,3 \%$ dos pacientes e continua sendo um sinto- ma importante no pós-operatório, seguida da anorexia (58,3\%), náuseas e vômitos (41,7\%) e diarreia (29,2\%).

Outros sintomas não relacionados ao pósoperatório de esofagectomia foram investigados e apresentaram porcentagens próximas a $42 \%$ para a dificuldade de mastigação e de $25 \%$ para a constipação.

Tabela 2 - Média, desvio padrão, valor mínimo e valor máximo dos resultados dos escores na SF-36 de acordo com o tipo histológico e com o tempo decorrido da operação.

\begin{tabular}{|c|c|c|c|c|c|c|c|c|c|c|c|}
\hline \multirow[b]{2}{*}{ Medida } & \multirow[b]{2}{*}{ média } & \multicolumn{3}{|c|}{ Adenocarcinoma $(n=10)$} & \multicolumn{7}{|c|}{ Carcinoma epidermóide $(n=14)$} \\
\hline & & $\begin{array}{l}\text { desvio } \\
\text { padrão }\end{array}$ & mediana & mínimo & máximo & média & $\begin{array}{l}\text { desvio } \\
\text { padrão }\end{array}$ & mediana & mínimo & máximo & valor-p \\
\hline Capacidade Funcional & 80,5 & 11,9 & 80,0 & 60,0 & 100,0 & 58,6 & 28,1 & 67,5 & 10,0 & 95,0 & 0,017 \\
\hline Limitação por aspectos Físicos & 57,5 & 39,2 & 62,5 & 0,0 & 100,0 & 41,1 & 41,2 & 25,0 & 0,0 & 100,0 & 0,3614 \\
\hline Dor & 54,5 & 21,6 & 61,5 & 10,0 & 84,0 & 57,1 & 28,1 & 62,0 & 20,0 & 100,0 & $0,8056^{*}$ \\
\hline Estado Geral de Saúde & 58,4 & 21,5 & 53,5 & 35,0 & 100,0 & 65,8 & 17,1 & 64,5 & 25,0 & 97,0 & 0,3591 * \\
\hline Vitalidade & 64,0 & 17,9 & 67,5 & 30,0 & 90,0 & 65,4 & 15,0 & 65,0 & 30,0 & 95,0 & 1,0000 \\
\hline Aspectos Sociais & 81,3 & 17,9 & 87,5 & 50,0 & 100,0 & 65,2 & 30,7 & 68,8 & 12,5 & 100,0 & 0,2570 \\
\hline $\begin{array}{l}\text { Limitação por Aspectos } \\
\text { Emocionais }\end{array}$ & 73,4 & 34,4 & 83,4 & 0,0 & 100,0 & 64,3 & 42,3 & 83,4 & 0,0 & 100,0 & 0,7317 \\
\hline Saúde Mental & 61,6 & 20,9 & 60,0 & 24,0 & 92,0 & 64,0 & 25,2 & 68,0 & 16,0 & 96,0 & $0,8076^{*}$ \\
\hline Tempo após operação meses & 19,2 & 20,4 & 10,4 & 5,6 & 64,0 & 33,4 & 43,6 & 11,9 & 5,0 & 132,0 & 0,8168 \\
\hline
\end{tabular}

teste de Mann-Whitney/ * teste $t$ de Student 
Tabela 3 - Variação de peso no pós-operatório de esofagectomia.

\begin{tabular}{|c|c|c|c|}
\hline & Média da Porcentagem & Mínimo $(\mathrm{Kg})$ & Máximo $(\mathrm{Kg})$ \\
\hline Adenocarcinoma & $16,84 \%$ & $-0,7$ & 45,9 \\
\hline CA Epidermoide & $8,54 \%$ & -2 & 22,9 \\
\hline Total & $12,00 \%$ & -2 & 45,9 \\
\hline
\end{tabular}

Tabela 4 - Questionário SF-36 de 24 doentes (58,8 anos) comparados aos de Aaronson et al. ${ }^{16}$ em uma população de saúde (4160 anos).

\begin{tabular}{|c|c|c|c|c|c|c|c|c|}
\hline Variável & $\begin{array}{r}\text { Tota } \\
\text { amostra }\end{array}$ & $\begin{array}{l}\text { tal da } \\
\text { a estudo }\end{array}$ & valor-p & $\begin{array}{c}\text { Adeno- } \\
\text { carcinoma }\end{array}$ & valor-p & Ca epidermóide & valor-p & $\begin{aligned} & \text { Amostra de } \\
& \text { Aaronsonet al. }{ }^{16}\end{aligned}$ \\
\hline Capacidade Funcional & 67,71 & $(\mathrm{sd} 24,98)$ & 0,003 & $80,5 \quad($ sd 11,89) & 0,473 & $58,57(\mathrm{sd} 28,11)$ & 0,0033 & $84,0($ sd 19,6) \\
\hline Limitação por aspectos Físicos & 47,92 & $(s d 40,32)$ & 0,023 & $(39,2)$ & 0,5430 & $41,1 \quad(s d 41,1)$ & 0,0270 & 74,5 (sd 36,8) \\
\hline Dor* & 56,04 & $(\mathrm{sd} 25,10)$ & 0,005 & $(21,58)$ & 0,0320 & $(28,09)$ & 0,0728 & $71,8(\mathrm{sd} 24,1)$ \\
\hline Estado Geral de Saúde & 62,71 & $($ sd 19,00$)$ & 0,046 & $58,4 \quad(s d 21,51)$ & 0,188 & $65,79($ sd 17,14) & 0,2329 & $69,7($ sd 20,6) \\
\hline Vitalidade & 64,79 & $(s d 15,91)$ & 0,371 & (sd 17,92) & 0,5410 & 65,36 (sd 15) & 0,4517 & $68,6(s d 20,2)$ \\
\hline Aspectos Sociais & 71,88 & $(\mathrm{sd} 26,90)$ & 0,056 & $81,25($ sd 17,92) & 0,731 & $65,18($ sd 15) & 0,0468 & $83,5(\operatorname{sd} 22,1)$ \\
\hline $\begin{array}{l}\text { Limitação por Aspectos } \\
\text { Emocionais }\end{array}$ & 68,07 & (sd 38,66) & 0,735 & $73,4 \quad(s d 34,4)$ & 0,844 & $64,3 \quad(\mathrm{sd} 42,3)$ & 0,5161 & $81,6($ sd 33,2) \\
\hline Saúde Mental* & 63 & $(\mathrm{sd} 23,03)$ & 0,013 & $61,6 \quad(s d 20,93)$ & 0,064 & $(s d 25,15)$ & 0,1081 & $75,6(s d 18,5)$ \\
\hline
\end{tabular}

teste de Mann-Whitney / * teste $t$ de Student

Tabela 5 - Associação de anorexia, disfagia, náuseas/vômitos, hábitos intestinais e tipo de câncer.

\begin{tabular}{|c|c|c|c|c|c|c|c|}
\hline & & & Ade & noma & Carcinoma & epidermoide & Valor-p \\
\hline & $\mathrm{n}$ & $\%$ & $\mathrm{n}$ & $\%$ & $\mathrm{n}$ & $\%$ & \\
\hline Anorexia & & & & & & & 0,6785 \\
\hline ausente & 10 & 41,7 & 5 & 50,0 & 5 & 35,7 & \\
\hline presente & 14 & 58,3 & 5 & 50,0 & 9 & 64,3 & \\
\hline Disfagia & & & & & & & 0,2721 \\
\hline ausente & 4 & 16,7 & 3 & 30,0 & 1 & 7,1 & \\
\hline presente & 20 & 83,3 & 7 & 70,0 & 13 & 92,9 & \\
\hline Mastigação & & & & & & & 0,2112 \\
\hline Boa & 14 & 58,3 & 4 & 40,0 & 10 & 71,4 & \\
\hline Ruim & 10 & 41,7 & 6 & 60,0 & 4 & 28,6 & \\
\hline Náusea/Vômito & & & & & & & 0,2112 \\
\hline ausente & 14 & 58,3 & 4 & 40,0 & 10 & 71,4 & \\
\hline presente & 10 & 41,7 & 6 & 60,0 & 4 & 28,6 & \\
\hline Hábito intestinal & & & & & & & 0,0713 \\
\hline constipação & 6 & 25,0 & 5 & 50,0 & 1 & 7,1 & \\
\hline diarreia & 7 & 29,2 & 2 & 20,0 & 5 & 35,7 & \\
\hline normal & 11 & 45,8 & 3 & 30,0 & 8 & 57,1 & \\
\hline
\end{tabular}

Teste exato de Fisher $(p>0,05)$

\section{DISCUSSÃO}

O foco deste estudo foi comparar a qualidade de vida de pacientes esofagectomizados por dois tipos histológicos de câncer esofágico: o Adenoca esofágico ou de cárdia e o CEC. A crescente frequência do Adenoca justifica esta preocupação ${ }^{17-20}$. Outra questão a considerar é o mais pronunciado aumento da sobrevivência dos pacientes com Adenoca esofágico comparado com os pacientes com CEC, em estudo realizado na Suécia, na década de noventa ${ }^{21}$.

O questionário SF-36 tem sido mundialmente utilizado e validado para avaliar doentes com doenças benignas e malignas, tanto no pré como no pós-operató- 
rio ${ }^{14,15,22}$. E foi muito útil nesta população estudada, pois $79 \%$ dos doentes entrevistados apresentavam algum grau de analfabetismo funcional. O emprego de uma escala visual que pudesse ser utilizada nestas situações para corroborar com os resultados apresentados, iria contribuir para obtenção das informações com mais facilidade. Além disso, por tratar-se de estudo transversal, durante a realização da pesquisa ocorreram óbitos inevitáveis, sendo esta dificuldade também relatada por outros autores $^{8}$

O ponto forte deste estudo foi a comparação dos resultados obtidos com os dados apresentados por Aaronson et al. ${ }^{16}$ para uma população saudável da Holanda, demonstrando diferenças significativas no domínio dor $(p=0,032)$ para o grupo Adenoca e, na capacidade funcional $(p=0,003)$, limitação por aspectos físicos $(p=0,027)$ e nos aspectos sociais $(p=0,0468)$ para o grupo CEC.

A qualidade de vida dos pacientes com Adenoca apresentou resultados semelhantes com o da população saudável, segundo os estudos De Boer et al. ${ }^{9}$, sendo exceção o domínio dor que difere da população saudável. Zieren et al. ${ }^{23}$, demonstraram que a qualidade de vida de doentes com CEC submetidos à esofagectomia mostrou ser prejudicada por limitações físicas.

Nesta pesquisa, as pontuações mais baixas foram encontradas nos domínios "Limitação por Aspectos Físicos" e "Limitação por Aspectos Emocionais". As pontuações mais baixas nestes domínios significam que o paciente apresenta problemas com o trabalho ou outra atividade diária em função da sua saúde física e emocional. Estas considerações foram confirmadas pela correlação encontrada entre os domínios "Saúde Mental" e "Limitação por Aspectos Emocionais" e "Limitação por Aspectos Físicos" e "Dor" para todos os pacientes, de modo geral e também segundo o tipo histológico. E o melhor resultado para o tipo histológico Adenoca no dominio "Capacidade Funcional" sugere que este paciente esteja mais apto do que o outro a realizar todo tipo de atividade física incluindo as mais vigorosas. Por outro lado, considerando que média do componente "Limitação por Aspectos Emocionais" foi 20 pontos maior do que a média da variável "Limitação por Aspectos Físicos", pode-se concluir que, após a esofagectomia, a condição física do paciente é uma dificuldade maior que a condição psicológica.

A análise da qualidade de vida dos pacientes neste estudo, em diferentes pontos transversais (de cinco meses a mais de oito anos de pós-operatório) não mostrou ter sido influenciada pelo tempo, ou seja não houve uma relação de dependência entre eles. Estes resultados estão em concordância com o estudo prospectivo de Djärv et al., incluindo pacientes esofagectomizados entre seis meses e três anos, sem diferença na qualidade de vida relacionada à saúde após a operação ${ }^{24}$.

A análise da dispersão (Figura 1) entre as variáveis qualidade de vida e o tempo decorrido de pósoperatório até o momento da entrevista, também não mostrou diferença estatística $(p>0,05)$, portanto, não influiu nos resultados finais da pesquisa. Entretanto, entendemos que este resultado deve ser interpretado com cautela por se tratar de uma amostra pequena, e a qualidade de vida é uma medida subjetiva e multidimensional e os resultados necessitam ser validados em uma amostra maior.

Não foi encontrada associação entre a qualidade de vida e disfagia, dificuldade de mastigação, anorexia, náuseas, vômitos, diarreia e constipação, embora estes dados tenham sido relatados nos dois tipos histológicos. A dificuldade de mastigação apresentada por $42 \%$ dos pacientes pode ser devido à ocorrência de $37 \%$ de idosos da amostra, em que a adequação de próteses dentárias e o cuidado com a saúde bucal poderia minimizar os problemas relatados.

Apesar de a esofagectomia por câncer de esôfago apresentar elevada morbidade operatória, é considerada como o tratamento padrão para pacientes com tumores ressecáveis e sem contra indicação clínica ${ }^{3,25}$. O conhecimento da qualidade de vida do paciente e dos pontos em que ela pode ser melhorada, certamente ajudará o paciente e a equipe de saúde a decidirem pelo melhor tratamento e de que forma enfrentarão a sua nova fase de vida.

É importante e indispensável que os doentes esofagectomizados recebam, no pós-operatório, orientações alimentares e comportamentais como parte do seu tratamento global. Neste sentido, a participação de equipe multiprofissional formada por nutricionistas, fisioterapeutas e psicólogos podem auxiliar o paciente na sua recuperação. O papel da família perante este enfrentamento contribuiu muito conforme as observações de Steinglass et al. ${ }^{26}$ na sua pesquisa da qualidade de vida em oncologia, destacando a necessidade de desenvolvimento de instrumentos para avaliação familiar.

Concluindo, este estudo mostrou melhor capacidade funcional para o grupo Adenoca em comparação com o grupo CEC e igualdade entre eles nos outros domínios da avaliação da qualidade de vida. A comparação destes grupos com uma população saudável mostra igualdade de vida desta população e o grupo Adenoca, com exceção de dor e piores resultados para a capacidade funcional, quando o grupo saudável é comparado com o CEC. Os pacientes dos dois grupos sofrem com disfagia, anorexia, dificuldade de mastigação, náuseas, vômitos, diarreia e constipação, que não se relacionaram com a qualidade de vida. 
Objective: To evaluate and compare the quality of life (QOL) of patients undergoing esophagectomy for treatment of adenocarcinoma of the esophagogastric junction and squamous cell carcinoma. Methods: We conducted a cross-sectional study in postoperative patients submitted to esophagectomy for adenocarcinoma of the esophagogastric junction (ACA) and squamous cell carcinoma (SCC), using the SF-36 questionnaire applied in 24 patients (10 ACAs and 14 SCCS), from the 5th months postoperatively, including clinical symptoms and weight change. Results: The assessment of QOL showed the best functional capacity $(p=0.018)$ in the ACA group. There was a correlation between the fields "mental health" and "Role of Emotions" $(p=0.003)$ and between "pain" and "physical aspects limitation" ( $p=0.003)$ in both histological types. Weight loss was greater in ACA (45.9 kg), with no significant difference between current BMI ( $p>0.66$ ). Dysphagia was reported by $83.3 \%$ of patients, anorexia by58.3\%, chewing difficulty by $42 \%$, nausea and vomiting by $41.7 \%$ and diarrhea by $29.2 \%$, with no correlation with QOL reported ( $p>0.05$ ). Conclusion: The highest score for functional capacity indicates that ACA patients were able to perform all types of physical activity, including the more demanding, at a higher level than patients with SCC. Some symptoms persisted postoperatively, but did not affect the quality of life of patients.

Key words: Adenocarcinoma. Squamous Cell Carcinoma. Quality of life. Esophagectomy. Swallowing disorders.

\section{REFERÊNCIAS}

1. Corley DA, Buffler PA. Oesophageal and gastric cardia adenocarcinomas: analysis of regional variation using the Cancer Incidence in Five Continents database. Int J Epidemiol. 2001;30(6):1415-25

2. Jankowski JA, Perry I, Harrison RF. Gastro-oesophageal cancer: death at the junction. BMJ. 2000;321(7259):463-4.

3. Tercioti Jr V, Lopes LR, Coelho Neto JS, Andreollo NA. New aspects of the neo-adjuvant therapy in esophageal squamous cell carcinoma. A review of medical literature. $A B C D$, Arq bras cir dig. 2009;22(1):33-40.

4. Steffen A, Schulze MB, Pischon T, Dietrich T, Molina E, Chirlaque $M D$, et al. Anthropometry and esophageal cancer risk in the European prospective investigation into cancer and nutrition. Cancer Epidemiol Biomarkers Prev. 2009;18(7):2079-89.

5. de Boer AG, van Lanschot JJ, van Sandick JW, Hulscher JB, Stalmeier PF, de Haes JC, et al. Quality of life after transhiatal compared with extended transthoracic resection for adenocarcinoma of the esophagus. J Clin Oncol. 2004;22(20):4202-8.

6. Cense HA, Visser MR, van Sandick JW, de Boer AG, Lamme B, Obertop $\mathrm{H}$, et al. Quality of life after colon interposition by necessity for esophageal cancer replacement. J Surg Oncol. 2004;88(1):328.

7. Blazeby JM, Farndon JR, Donovan J, Alderson D. A prospective longitudinal study examining the quality of life of patients with esophageal carcinoma. Cancer. 2000;88(8):1781-7.

8. Rosmolen WD, Boer KR, de Leeuw RJ, Gamel CJ, van Berge Henegouwen MI, Bergman JJ, et al. Quality of life and fear of cancer recurrence after endoscopic and surgical treatment for early neoplasia in Barrett's esophagus. Endoscopy. 2010;42(7):52531.

9. De Boer AG, Genovesi PI, Sprangers MA, Van Sandick JW, Obertop $\mathrm{H}$, Van Lanschot JJ. Quality of life in long-term survivors after curative transhiatal oesophagectomy for oesophageal carcinoma. Br J Surg. 2000;87(12):1716-21.

10. McLarty AJ, Deschamps C, Trastek VF, Allen MS, Pairolero PC, Harmsen WS. Esophageal resection for cancer of the esophagus: long- term function and quality of life. Ann Thorac Surg. 1997;63(6):1568-72.

11. Kirby JD. Quality of life after oesophagectomy: the patients' perspective. Dis Esophagus. 1999;12(3):168-71.

12. OMS. El estado físico: uso e interpretación de la antropometria. Informe de un comité de Expertos de la OMS. Serie de informes técnicos 854. Genebra: OMS; 1993.
13. Lipschitz DA. Screening for nutritional status in the elderly. Prim Care. 1994;21(1): 55-67.

14. Ware JE Jr, Sherbourne CD. The MOS 36-item short-form health survey (SF-36). I. Conceptual framework and item selection. Med Care. 1992;30(6):473-83.

15. Ciconelli RM, Ferraz MB, Santos W, Meinão I, Quaresma MR Tradução para a lingua portuguesa e validação do questionário genérico de avaliação de qualidade de vida SF-36 (Brasil SF-36). Rev Bras Reumatol. 1999;39(3):143-50.

16. Aaronson NK, Muller M, Cohen PD, Essink-Bot ML, Fekkes M, Sanderman $\mathrm{R}$, et al. Translation, validation, and norming of the Dutch language version of the SF-36 Health Survey in community and chronic disease populations. J Clin Epidemiol. 1998;51(11):105568.

17. Jung KW, Talley NJ, Romero Y, Katzka DA, Schleck CD, Zinsmeister $A R$, et al. Epidemiology and natural history of intestinal metaplasia of the gastroesophageal junction and Barrett's esophagus: a population-based study. Am J Gastroenterol. 2011;106(8):144755

18. Modena SF, Meirelles LR, Araujo MR. Are the nitrites important in a Genesis of the adenocarcinoma associated to the Barrett esophagus? ABCD, Arq Bras Cir Dig. 2008;21(3):124-9.

19. Cooper GS, Yuan Z, Chak A, Rimm AA. Association of prediagnosis endoscopy with stage and survival in adenocarcinoma of the esophagus and gastric cardia. Cancer. 2002;95(1):32-8.

20. Schembre D, Arai A, Levy S, Farrel-Ross M, Low D. Quality of life esophagectomy and endoscopic therapy for Barrett's esophagus with dysplasia. Dis Esophagus. 2010;23(6):458-64.

21. Sundelöf $M$, Ye W, Dickman PW, Lagergren J. Improved survival in both histologic types of oesophageal cancer in Sweden. Int J Cancer. 2002;99(5):751-4

22. Brown LF, Kroenke K, Theobald DE, Wu J. Comparison of SF-36 vitality scale and Fatigue Symptom Inventory in assessing cancerrelated fatigue. 2011;19(8):1255-9.

23. Zieren HU, Jacobi CA, Zieren J, Müller JM. Quality of life following resection of oesophageal carcinoma. Br J Surg. 1996;83(12):17725.

24. Djärv T, Lagergren J, Blazeby JM, Lagergren P. Long-term healthrelated quality of life following surgery for oesophageal cancer. $\mathrm{Br}$ J Surg. 2008;95(9):1121-6.

25. Tercioti Júnior V, Lopes LR, Coelho Neto Jde S, Carvalheira JB, Andreollo NA. Eficácia local e complicações da terapêutica neoadjuvante no carcinoma epidermoide: radioterapia versus radioterapia associada à quimioterapia. Rev Col Bras Cir. 2011;38(4):227-34 
26. Steinglass $P$, Ostroff JS, Steinglass AS. Multiple family groups for adult cancer survivors and their families: a 1-day workshop model. Fam Process. 2011;50(3):393-409.

Recebido em 25/05/2012

Aceito para publicação em 30/07/2012

Conflito de interesse: nenhum

Fonte de financiamento: nenhuma
Como citar este artigo:

Pereira MR, Lopes LR, Andreollo NA. Qualidade de vida de doentes esofagectomizados: adenocarcinoma versus carcinoma epidermoide. Rev Col Bras Cir. [periódico na Internet] 2013;40(1). Disponível em URL: http://www.scielo.br/rcbc

\section{Endereço para correspondência:}

Nelson Adami Andreollo

E-mail: nandreollo@hotmail.com 